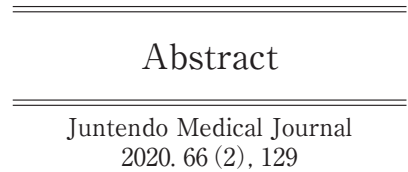

\section{The Characterization of Pathogenic and Non-pathogenic HLA-B27 Protein}

\author{
KosUKe MATSUZAKI* \\ *Department of Immunology, Juntendo University Graduate School of Medicine, Tokyo, Japan
}

\begin{abstract}
Ankylosing spondylitis (AS) is characterized by chronic inflammation of the axial and peripheral joints and ligamentous attachments. The major histocompatibility complex (MHC) haplotype HLA-B27 has the strongest genetic association with the disease. Among these subtypes of HLA-B27, B*2702, B*2703, B*2704, B*2705 and B*2710 are reported to significantly increase risk, whereas B*2706 and B*2709 are not associated with disease. To date, three major hypothesis have been raised. The first one is that the arthritogenic peptide presented by HLA-B27 activates $\mathrm{CD} 8^{+} \mathrm{T}$ cells to cause inflammatory arthritis. The second theory is that misfolding of HLA-B27 in the endoplasmic reticulum leads to endoplasmic reticulum stress resulting in the activation of unfolded protein responses (UPRs) and the upregulation of IL-23 in dendritic cells. The third theory is that the HLA-B27 has an ability to aberrantly fold to form homodimers and this homodimer can be recognized by killer-immunoglobulin-like receptors. Despite these theories have been studied, the pathogenic role of HLA-B27 still remains unclear.

In the present study, in order to clarify the function of HLA-B27 at the cellular level, we established human-derived B cell line C1R stably expressing pathogenic HLA-B27 subtypes (B*2704 or B*2705) or non-pathogenic subtype (B*2706). We examined the localization of HLA-B27 using confocal microscopy. We also investigated their associated molecules using Liquid chromatography-tandem mass spectrometry (LC-MS/MS).

Confocal microscopic analysis demonstrated that either B*2704 or B*2705 form smaller uneven multiple clusters on the cell surface compared to that of B*2706 which form a single large dense cluster on the cell surface. LC-MS/MS analysis showed that the molecules which bind to both B*2704 and B*2705 but not B*2706 were Target of Myb protein 1 (Tom1) and MHC class I molecules. Tom 1 is an adaptor protein required for the maturation of autophagosomes and their fusion with lysosomes. Tom 1 also participates in immune receptor recycling and Toll-like receptor signaling. Therefore, pathogenic HLA-B27 might have some effect on immune receptor or Toll- like receptor- mediated signals by the alteration of intracellular vesicle trafficking. The other associated molecule was MHC class I molecules heterodimer with other MHC class I molecules in addition to HLA-B27 or $32-$ microgloblin. Although the direct evidence of their association should be demonstrated in the future studies, a new type of heterodimer may have some effect on immune responses.
\end{abstract}

To summarize these results, the AS-sensitive subtype and insensitive subtypes of HLA-B27 differed in quantitative, qualitative, and diversity. It was also speculated that they might lead to the pathogenesis of AS.

Key words: ankylosing spondylitis (AS), HLA-B27, target of Myb protein 1 (Tom1)

Kosuke Matsuzaki

Department of Immunology, Juntendo University Graduate School of Medicine

2-1-1 Hongo, Bunkyo-ku, Tokyo 113-8421, Japan

TEL: +81-3-3813-3111 E-mail: med2114103@stud.juntendo.ac.jp

Research of the 2nd Alumni Scientific Award for Medical Student, Juntendo University School of Medicine

〔Received Aug. 26, 2019〕〔Accepted Mar. 9, 2020〕

Copyright (C) 2020 The Juntendo Medical Society. This is an open access article distributed under the terms of Creative Commons Attribution License (CC BY), which permits unrestricted use, distribution, and reproduction in any medium, provided the original source is properly credited. doi: 10.14789/jmj.2020.66.JMJ19-A03 